\title{
Análisis de los Jueces de Paz en Neiva (Colombia)
}

Semillero Estudios Constitucionales Mirfelly Rocío Velandia Bermeo, Johnatan Otero Devia, Maicol Aníbal Quintero Montes, Jaime Arturo Camero, Ivan Ariel Osorio Cabrera, Katherin Torres Posada.

Docente Tutora - Lucy Miriana Núñez Benavidez Grupo Nuevas Visiones del Derecho / Universidad Surcolombiana

Articulo recibido: 08/02/2009 Aprobado: 10/06/2009

\section{RESUMEN}

La jurisdicción especial de paz es una figura reciente y novedosa en nuestro ordenamiento jurídico, creado por el Constituyente de 1991, quien buscó instituir una figura próxima a la comunidad, de origen popular que brindara una solución equitativa a las diferencias existentes entre las personas que forman parte de dicha colectividad, y con ello se descongestionara la jurisdicción ordinaria. En la actualidad, después de dieciocho años de haberse creado esta figura, en algunas partes del país su proceso de implementación aún no se hallevado a cabalidad.

El presente artículo se encuentra dividido en dos partes, en un primer momento, se analizaran los diferentes pronunciamientos que ha efectuado; la Corte Constitucional, en temas tan importantes como la naturaleza jurídica de esta jurisdicción, la procedencia de la acción de tutela contra fallos de los jueces de paz y la falta de remuneración de los jueces de paz.

Posteriormente, se expone la forma en que se desarrolló el proceso de implementación de esta figura en la ciudad de Neiva y los resultados encontrados dentro de la investigación realizada.

\section{PALABRAS CLAVE}

Jurisdicción especial de paz, implementación, fallos en equidad, conciliación, justicia comunitaria.

\section{ABSTRACT}

The special jurisdiction of peace is a recent and novel set in our legal system, created by the 1991 constituent, who sought to establish a figure close to the community, grassroots that would provide an equitable solution to the differences between people who are part of that group, and thereby relieve the ordinary courts. Today, after eighteen years this figure has been created in some parts of the country the implementation process has not yet been fully implemented.

This article is divided into two parts; at first, we analyze the various pronouncements that made by the Constitutional Court, on such important issues as the legal nature of this jurisdiction, the merits of the action for protection against failures Justices of the Peace, and the lack of compensation for justices of the peace. Subsequently, we illustrate the way in which developed the process of implementing this figure in the city of Neiva and findings in the investigation.

\section{KEYWORDS}

Special peace jurisdiction, implementation, equity failures, reconciliation, community justice. 


\section{ANÁLISIS JURISPRUDENCIAL}

Para tener una mejor ilustración respecto a la justicia de paz, es necesario examinar, cúal ha sido la postura que ha asumido la Corte Constitucional en temas tan relevantes como los que se exponen a continuación:

\subsection{Naturaleza jurídica de la justicia de paz:}

Para la Corte, la creación de la jurisdicción especial trasciende el simple propósito de descongestión de los despachos judiciales para atender de manera más eficiente las necesidades ciudadanas de justicia; por ello su establecimiento involucra un replanteamiento de la relación entre Estado y ciudadano desde dos puntos de vista, tal y como lo manifestó la Corte en sentencia C-536 de 1995, con ponencia del Magistrado Jaime Córdoba Triviño:

"Tanto desde la perspectiva genérica de la consagración del Estado Social de Derecho en tanto fórmula política fundamental, como desde el punto de vista especifico de la introducción de una serie de mecanismos alternativos a la justicia formal para la resolución de los conflictos sociales, fue deseo del constituyente consolidar un modelo nuevo de interacción entre la ciudadanía y el poder público, que -entre otras- fomentara un acercamiento progresivo de los mecanismos formales de promoción de la convivencia a las realidades sociales en las que habrian de operar."

De igual manera, en ese mismo pronunciamiento estableció que dicha creación fue prevista como un canal para que el ciudadano común participe, "en virtud de sus calidades personales y su reconocimiento comunitario, en la función pública de administrar justicia, jugando así un rol complementario al que asignó la Carta a las demás autoridades y particulares que participan de dicho cometido estatal".

La jurisprudencia del alto tribunal ha definido la naturaleza jurídica de la justicia de paz, estableciendo que se inscribe dentro del concepto de democracia participativa, puesto que permite que el ciudadano intervenga en las funciones que pertenecen al Estado.

En cuanto a las decisiones que profieren los jueces de paz, así lo ha señalado la Corte, en ese mismo pronunciamiento:

"Sus decisiones escapan el ámbito de lo jurídico, no deben fundamentarse en esa labor única del juez ordinario de fallar conforme a lo que establece la ley. A través de la equidad, entonces, se pretende también administrar justicia pero, por mandato constitucional, en aquellos eventos de menor importancia en que el rigor de la ley no resulta aplicable o no prevé una situación específica. No se busca, por ende, reemplazar las funciones del aparato estatal encargado de dirimir en derecho los conflictos existentes sino, por el contrario, complementarlo".

\subsection{La labor ad-honorem de los jueces de paz:}

Este tema ha generado bastante controversia y posiciones divergentes, no solo entre los jueces de paz, sino entre los académicos; ante ello la Corte en sentencia C-103 de 2004 ha manifestado que:

"El Congreso de la República, optó por un modelo de jueces de paz no remunerados, por lo cual, no se puede afirmar por esa sola decisión que desconoció la Carta Política, además, la misma Ley 497 de 1999, permite que un juez de paz desempeñe otra actividad en el sector público o privado para así obtener libremente los ingresos requeridos para su sustento; además esta labor es desempeñada por quien de manera libre y voluntaria asume una carga adicional, a sabiendas de su no remuneración".

Es claro que el constituyente consagró algunos elementos del servicio público en el artículo 122 Superior. Alli dijo: "No habrá empleo público que no tenga funciones detalladas en ley o reglamento y para proveer los de carácter remunerado se requiere que estén contemplados en la respectiva planta y previstos sus emolumentos en el presupuesto correspondiente". Además, el artículo 
13 de la Carta Fundamental, permite al legislador establecer diferenciaciones entre personas que se encuentran en circunstancias objetivamente distintas, es decir:

"Bien puede el legislador, en ejercicio de la potestad amplia que le otorgó expresamente el Constituyente en la materia, establecer condiciones para el ejercicio del cargo de juez de paz que no son iguales a las de los demás servidores públicos o particulares que ejercen funciones de naturaleza junisdiccional."

\section{3 ¿Es inconstitucional la competencia que} tiene los jueces de paz para conocer casos de violencia intrafamiliar?

Esta cuestión es analizada en sentencia C-059 de 2005 , donde se examina la constitucionalidad del parágrafo $1^{\circ}$ del artículo $1^{\circ}$ de la Ley 575 de 2000 en el cual se les confiere competencia para conocer de los casos de violencia intrafamiliar, a la jurisdicción de paz y a los conciliadores en equidad.

Al respecto, sostuvo la Corte que la Constitución Política, en su artículo 247, encargó la creación de dicha jurisdicción para resolver en equidad conflictos individuales y comunitarios, "sin que para el efecto los hubiere excluido de algunos asuntos en particular".

En efecto, tratándose del fenómeno de la violencia intrafamiliar el constituyente no descartó la posibilidad de que fuera enfrentado con instrumentos distintos al represivo, como lo es la intervención mediadora de los jueces de paz y de los conciliadores en equidad, que constituyen mecanismos alternativos de solución de conflictos, cuyo empleo está plenamente autorizado por la Carta Política para alcanzar los objetivos fundamentales de la paz y el sosiego de la comunidad. Igualmente expreso que:

"No es cierto que al asignarle competencia a los jueces de paz y a los conciliadores en equidad para que conozcan casos de violencia intrafamiliar la norma acusada viole la Carta Política; por el contrario, lo que se hace es efectivizar los mandatos superiores relacionados con la garantía de protección integral a la familia, y aquellos atinentes a la pronta y efectiva administración de justicia, dándole para ello desarrollo a los mecanismos alternativos de solución de conflictos y propendiendo por el cumplimiento de los fines del Estado social de derecho."

Por tal razón, los jueces de paz, son competentes para conocer de casos de violencia intrafamiliar, por ser precisamente ésta figura, el mecanismo idóneo para alcanzar los objetivos superiores de la protección integral de la familia e igualmente la participación de la comunidad en los problemas que los afectan.

\subsection{Procedencia de la acción de tutela contra los fallos en equidad:}

La Corte en sentencia T- 796 de 2007, abordo el tema de la acción de tutela en contra las decisiones de los jueces de paz. Se trataba de una persona que celebró contrato de arrendamiento con el supuesto representante de una Junta de Acción Comunal (JAC). Los demás miembros de la JAC le pusieron de presente a la persona, que el supuesto arrendador no tenía facultades de representación, y la instaron a concurrir ante un juez de paz para dirimir la controversia. Dado que se agotó la etapa conciliatoria sin acuerdo, la decisión del juez de paz fue la de que la arrendataria debía restituir el inmueble a la Junta. Recurrida la decisión, fue confirmada por los jueces de reconsideración.

La persona despojada interpuso acción de tutela contra las decisiones, puesto que estimó que con esta actuación de los jueces de paz, le vulneraron los derechos fundamentales a la defensa y al debido proceso, pues sostuvo que se desconoció un acto jurídico como es el contrato de arrendamiento, y no se le ha iniciado ningún proceso de restitución. Considera que el conflicto no podía ser definido por los jueces de paz.

Frente a este caso, destaca la Corte que la labor del juez de paz es una labor conciliadora, pues busca una solución que, además de justa, pueda ser concertada; sin embargo, bajo el reconocimiento de que no todos los conflictos pueden ser resueltos 
mediante un acuerdo amigable, el juez de paz está investido de la capacidad de fallar, de resolver por vía de autoridad el conflicto que se lleva a su conocimiento, de forma que sus decisiones cuentan con fuerza obligatoria y definitoria. Indicó además que:

"El papel de los jueces de paz no se circunscribe a ser simples operadores judiciales que apoyan la descongestión de los despachos judiciales, sino principalmente facilitadores de procesos de aprendizaje comunitario, porque lo más importante de esta jurisdicción es la posibilidad que ella brinda para que las comunidades construyan en forma participativa unos ideales de lo justo, y desarrollen también en forma integrada y armónica habilidades de resolución pacífica de conflictos, a partir del interés que suscitan los problemas sociales cotidianos".

Con base en los criterios de equidad, sus decisiones se deben basar en la aplicación del recto criterio que lleve a la solución justa y proporcionada de los conflictos humanos, es decir, la actuación de los jueces reflejará las convicciones de la comunidad acerca de lo que es justo con miras a garantizar la convivencia pacífica, respetando los preceptos constitucionales y el debido proceso previsto en la propia normatividad que la establece; además su actuar deberá ceñirse a los principios que orientan la jurisdicción de paz: equidad, gratuidad, oralidad, garantía de derechos, autonomía e independencia.

Con este pronunciamiento, la Corte Constitucional marca un precedente, frente a la procedibilidad de la acción de tutela contra las decisiones que profieren en equidad los jueces de paz, entendiendo que ésta no puede ser analizada bajo el prisma de las reglas de procedencia de la acción de tutela contra decisiones proferidas por los jueces que actúan en derecho ya que la tutela excepcional contra decisiones judiciales, se funda en que al juez que administra justicia formal se le exige en esta tarea, el sometimiento a la Constitución y a la ley; además, porque este se ha concebido como un mecanismo de defensa no solamente frente a aquellos eventos en que el juez impone de manera grosera y burda su voluntad sobre el ordenamiento jurídico, sino frente a situaciones en que se aparta de los precedentes sin una debida argumentación, y cuando su discrecionalidad interpretativa se desborda en perjuicio de los derechos fundamentales de los asociados. "Las sentencias que profiere constituyen supuestos específicos de aplicación del derecho, cuya legitimidad viene reconocida desde luego, por la realización de fines estatales y, en particular, por la garantía de los derechos constitucionales".

Lo anterior no significa que un juez de paz posea atribuciones ilimitadas, ya que "el umbral para el ejercicio autónomo e independiente de su labor de administrar justicia en equidad lo determina la Constitución y la ley y en particular los derechos fundamentales de los intervinientes en la actuación, así como de los terceros afectados y en ese marco se debe efectuar el control constitucional sobre sus decisiones." 1

Finalmente, la Corte decidió no acceder a las pretensiones de la demandante, ya que estimó, que los jueces de paz decidieron el conflicto aplicando criterios de equidad soportados en la prueba documental que le fue aportada por la partes, y en su percepción directa de los hechos obtenida mediante inspección judicial que practicó en el inmueble que originó la disputa. El consentimiento de la entonces arrendataria para someter su conflicto a la justicia de paz aparece documentado sin que milite prueba que lo desvirtúe, y el procedimiento se ciñó a los causes previstos en la Ley 497 de 1999, por lo que no determino el Tribunal Constitucional vulneración alguna a los derechos fundamentales al debido proceso y a la defensa que invoca la demandante.

La jurisdicción especial de paz en palabras de la Corte Constitucional, "fué creada como una vía expedita para la resolución de conflictos individuales y comunitarios. En ella subyace el deseo de construir la paz desde lo cotidiano, de

${ }^{1}$ En el mismo sentido, ver sentencia T- 809 de 2008. M.P. Manuel José Cepeda. 
alcanzar la convivencia pacífica a partir de una justicia diferente a la estatal, tanto por su origen y el perfil de los operadores, como por los fines y los mecanismos propuestos para su ejecución", y fué voluntad del mismo constituyente otorgar al legislador, la facultad de regular la jurisdicción de paz en cuanto a su designación y a su funcionamiento, razón por la cual, con la expedición de la Ley 497 de 1999, el Congreso de la República, estableció condiciones para el ejercicio del cargo de juez de paz que no son iguales a las de los demás servidores públicos o particulares que ejercen funciones de naturaleza jurisdiccional.

Así mismo el legislador, tenía abierta la posibilidad de establecer que el cargo de juez de paz fuera remunerado por medio de ésta ley. Optó por la decisión contraria, en atención a la naturaleza del cargo y a las finalidades que se pretenden con su creación, debido a que no ostentan la calidad de servidores públicos como tal, sino que son particulares con facultades para administrar justicia.

La gratuidad es uno de los principios rectores de ésta figura de conformidad con la Ley 497 de 1999, de tal manera que el remunerar a quien desempeña esta función, implicaría un cambio notable dentro de dicha ley; se haría necesario reestructurar los principios que la rigen, lo que conllevaría a una desnaturalización de la figura, perdiendo el sentido social, de manera, que quien aspire a desempeñarse como juez de paz, no lo haría con la intención de prestar un servicio a la comunidad, sino como una forma de vida, lo cual iría en contra del espíritu del legislador al crear la jurisdicción de paz como una alternativa de solución de conflictos.

Sin embargo esto no es óbice para que las entidades encargadas de velar por el adecuado y eficaz funcionamiento de la justicia de paz, como es el caso del Consejo Seccional de la Judicatura y de la Alcaldía Municipal, no brinden los elementos y recursos necesarios para desarrollar tan importante función dentro de una comunidad, en aras de encontrar una salida a los problemas para resolverlos de la mejor manera, impartiendo así justicia, que tanta falta le hace a la sociedad.

\section{PROCESO DE IMPLEMENTACIÓN DE IA JUSTICIA DE PAZ EN NEIVA}

El proceso de implementación de los jueces de paz en la ciudad de Neiva, adelantado en el 2004 por la Alcaldía Municipal en convenio interadministrativo con la Universidad Surcolombiana, se desarrolló a través de cuatro estrategias:

2.1 Capacitación: Esta etapa se promovió a partir de talleres dingidos a los candidatos a ejercer la función de juez de paz y de reconsideración, a grupos de vecinos organizados y agremiaciones de los barrios y corregimientos del municipio. Se utilizó la cartilla "jueces de paz para Neiva", con el fin de incentivar en la comunidad la participación de las primeras elecciones que se llevarian a cabo en la ciudad; así mismo, se realizaron reuniones comunitanias, que tenían como propósito divulgar y sensibilizar a los participantes del impacto y los beneficios de la jurisdicción de paz; en ésta primera etapa, se evidenciaron algunos prejuicios por parte de la comunidad, con lo que se ponía en riesgo la pérdida de respaldo y credibilidad por parte de la población, precisamente con ésta primera estrategia se pretendió desvirtuar éste tipo de pensamientos.

2.2 Difusión y promoción: se ejecutó a través obras de teatro en diferentes lugares de la ciudad, con lo que se buscó generar un impacto de interés en la población; igualmente se repartieron plegables en los sitios más concurnidos, con el propósito de forjar un alto grado de participación de la comunidad en general, y encontrar un respaldo popular a la justicia de paz en la ciudad.

De igual forma, y con miras a dar a conocer la figura del juez de paz en toda la ciudad, se realizaron diversas exposiciones en diferentes lugares dirigidas a la población en general, y se acudió a los diferentes medios de comunicación de la región, gracias a los cuales se logró no solo dar a conocer la figura sino que también se mantuvo informada a la población de todas las actividades a 
desarrollarse con la justicia de paz, específicamente lo relacionado a la capacitación y al proceso de inscripción de los candidatos, y posteriormente a la elección de éstos servidores; de la misma forma en los puestos de votación y en el centro de Neiva se expusieron pasacalles con mensajes del día electoral.

\subsection{Apoyo pedagógico a la campaña de los} jueces de paz y de reconsideración: El desarrollo de esta estrategia por parte del equipo ejecutor del proyecto se basó en aspectos tales como: planeación de las actividades correspondientes al proceso de campaña electoral, acompañamiento y asesoría a los candidatos de las comunas, y entrega de material de apoyo (plegables, cartillas, afiches e información de la comuna). Se realizaron además reuniones comunitarias donde se hizo la presentación oficial de los candidatos, se ofrecieron exposiciones sobre la justicia de paz y se discutieron los beneficios de la figura y la importancia de la convivencia pacífica en la ciudad.

Finalmente, se ejecutó la estrategia de seguimiento y formación, donde periódicamente el equipo coordinador se reunió con los electos jueces de paz y de reconsideración, con el fin de determinar los contenidos temáticos indispensables para su funcionamiento, los planes estratégicos y actividades que desean desarrollar de manera individual o colectiva en sus comunas y en la ciudad.

A su vez, con esta estrategia se orientó la labor del juez de paz y de reconsideración, para lo cual se realizaron actividades tendientes a estructurar los contenidos y alcances de la justicia de paz, fundamentados en el artículo 247 de la Constitución Política de 1991 y la Ley 497 de 1999.

De igual manera, los jueces recibieron capacitación en diversos temas tales como los derechos humanos, informática básica, desarrollo humano, democracia, liderazgo juvenil, resolución de conflictos y participación comunitaria, entre otras.

Sin embargo, durante esta etapa, se presentaron múltiples factores que representaron limitaciones en su implementación, tales como la falta de disposición de tiempo, la ausencia de espacios para la realización de las reuniones comunitarias, la falta de herramientas y los prejuicios que surgieron en la comunidad y en los mismos candidatos acerca de la figura, y por último, se evidenciaron los vicios tradicionales propios de una campaña electoral. A pesar de esto, dicho proceso cumplió con su objetivo, pues gracias a éste la ciudad, le apostó a una solución pacifica de los conflictos, a través de ésta jurisdicción especial.

\section{EL PROBLEMA DE INVESTIGACIÓN}

En Neiva no existen estudios que nos den a conocer la manera como funciona esta jurisdicción en los últimos años, por tal razón, surgen ciertos interrogantes: ¿Cómo funciona la jurisdicción de paz?, ¿Cómo se han desempeñado los jueces de paz, en ejercicio de sus funciones? ¿Cuál fue el proceso de implementación en la ciudad?, ¿Cuáles son sus principales problemas?, ¿Cuál es el concepto que tienen los jueces de la equidad? ¿Cómo han sido los procesos de capacitación y formación que recibieron los jueces de paz? Por lo que resulta necesario responder al siguiente interrogante que vislumbrara la realidad de los diferentes aspectos del funcionamiento de la Justicia de Paz en Neiva dentro del periodo objeto de nuestra investigación:

¿Cómo ha sido la implementación, funcionamiento y los resultados de los jueces de paz en Neiva durante el período 2004-2007?

\section{DISEÑO METODOLÓGICO \\ DE LA INVESTIGACIÓN}

Este tipo de investigación es temática - empírica con un enfoque socio jurídico que pretende describir los elementos cuantitativos y cualitativos de la justicia de paz. Igualmente, evalúa las diversas facetas del proceso de esta jurisdicción desde el instante en que las personas acuden ante el juez de paz, hasta la etapa en que éste emite un fallo. 
En primer lugar, de una fundamentación teónica, desarrollada a partir de un análisis junisprudencial y bibliográfico de diversos textos que prevén la implementación de este mecanismo de solución de conflictos a nivel nacional yregional.

Posteriormente, se utilizaron los siguientes elementos para la recolección de información:

De carácter cualitativo: Entrevista semiestructurada a los jueces de paz y de reconsideración. Observación no participante, realizando un acompañamiento a los jueces de paz en su lugar de atención y sesiones de conciliación. Análisis documental de las actas de conciliación y los fallos en equidad que profieren los jueces de paz y de la capacitación impartida del Consejo Superior de la Judicatura, Seccional Huila.

De carácter cuantitativo: Encuestas cerradas de tipo aleatorio a la comunidad con el fin de establecer el grado de conocimiento y aceptación de esta figura y estadísticas de los tipos de conflicto más frecuentes.

\section{RESULTADOS}

En primer lugar, se realizó la tabulación que corresponde al formato trimestral que deben diligenciar los jueces de paz, ante el Consejo Seccional de la Judicatura, puesto que es el único instrumento que permite determinar la cantidad de casos que manejan estos actores comunitarios; en dicho formato se hace una relación de la cantidad de procesos tratados en ese período, el tipo de conflicto y la manera de terminación.

De dicho trabajo se encontró que durante el año 2006 se presentaron un total de 155 casos. Los conflictos más frecuentes correspondían en un $18 \%$ a temas relacionados con la convivencia familiar, representando este el más alto porcentaje; seguido de invasión de espacio público con un $13 \%$ e injuria y calumnia con un $12 \%$ (ver tabla 1 ). El $30 \%$ de conflictos se presentaron en la comuna 10 , seguido de la comuna 6 con un $19 \%$ y el Corregimiento El Caguán con un 10\% (tabla 2).

\section{Tabla 1. Tipo de Conflicto}

TIPO DE CONFLICTO

Convivencia Familiar

Invasión de espacio público

Injuria y calumnia

$\begin{array}{cc}\text { TOTAL DE CASOS } & \text { PORCENTAJE } \\ 25 & 18 \% \\ 18 & 13 \% \\ 17 & 12 \%\end{array}$

$\begin{array}{cc}\text { TOTAL DE CASOS } & \text { PORCENTAJE } \\ 46 & 30 \% \\ 30 & 19 \% \\ 16 & 10 \%\end{array}$

Tabla 2. Comunas con mayor frecuencia de casos

COMUNAS CON MAS NUMEROS DE CASOS

Comuna 10

Comuna 6

Corregimiento El Caguán.

En el año 2007 se registraron un total de 183 casos, de ellos el conflicto más reiterativo fué el de mal manejo de mascotas con un $22 \%$, seguido de injuria y calumnia con un $20 \%$ y convivencia familiar con un $13 \%$ (ver tabla 3). La comuna que presentó mayor índice de procesos fué la comuna 9 con un $52 \%$, luego la comuna 10 con un $15 \%$, seguido de la comuna 6 con un $12 \%$ (tabla 4 ).

Tabla 3. Tipo de conflicto

TIPO DE CONFLICTO

Mal manejo de mascotas

Injuria y calumnia

Convivencia familiar

$\begin{array}{cc}\text { TOTAL DE CASOS } & \text { PORCENTAJE } \\ 40 & 22 \% \\ 36 & 20 \% \\ 23 & 13 \%\end{array}$

Tabla 4. Comuna con más número de casos

COMUNAS CON MAS NUMERO DE CASOS

Comuna 9

Comuna 10

TOTAL DE CASOS

PORCENTAJE

95

27

$52 \%$

$15 \%$

21 
De los casos que conocieron los jueces de paz en el año 2006 se encontró que el $99 \%$ terminó con conciliación y tan sólo el 1\% con fallo en equidad; de igual manera, durante el año 2007 el $98 \%$ de los procesos se resolvieron por conciliación mientras que el $1 \%$ fué remitido y el $1 \%$ restante culminó con desistimiento. (Ver tabla 5).

Tabla 5. Forma de terminación del proceso

FORMA DE TERMINACIÓN DEL PROCESO

Acta de conciliación

Fallo en equidad

Desistimiento

Remisión

AÑo 2006
154
1
0
0

AÑO 2007

181

0

1

1

Los resultados que se obtuvieron de las 844 encuestas aplicadas a la comunidad, de manera aleatoria, se obtuvo que el $75 \%$ de los encuestados, no tienen conocimiento de la jurisdicción especial de paz y tan sólo el 35\% conoce de ésta (ver tabla 6). Quienes afirmaron conocer esta jurisdicción, manifestaron que se enteraron de la existencia de ello por los medios de comunicación en un $51.6 \%$, por medio de amistades se enteraron el $26 \%$, mientras que el $8.6 \%$ expresó que se enteró por familiares y tan sólo el $5.3 \%$ sabe de esta jurisdicción por quedar cerca a la residencia (ver tabla 7).

Tabla 6. ¿Tiene conocimiento de la jurisdicción especial de paz?

$\begin{array}{lc}\text { ¿TIENE CONOCIMIENTO DE LA JURISDICCIÓN ESPECIAL DE PAZ? } & \text { TOTAL } \\ \text { NO } & 637 \\ \text { SI } & 207 \\ \text { TOTAL ENCUESTADOS } & 844\end{array}$

Tabla 7. ¿Cómo se enteró de la existencia de la justicia de paz?

$\begin{array}{lc}\text { ¿CÓMO SE ENTERó DE LA EXISTENCIA DE LA JUSTICIA DE PAZ? } & \text { TOTAL } \\ \text { Medios de Comunicación } & 110 \\ \text { Amigos } & 54 \\ \text { Familiares } & 18 \\ \text { Funcionario público } & 14 \\ \text { Queda Cerca a la Residencia } & 11 \\ \text { TOTAL } & 207\end{array}$

De los 207 encuestados que afirmaron conocer la jurisdicción especial de paz, el $20.7 \%$ manifestó haber acudido durante el último año a dicha jurisdicción para resolver algún conflicto; mientras que el $79.3 \%$ expresó no haber acudido a ella porque no han tenido problemas ( $78 \%$ ) o porque acuden a otros medios de solución de conflicto (22\%). (Ver tabla 8).

Tabla 8. ¿Ha acudido a la jurisdicción de paz?

$\begin{array}{lcc}\text { ¿HA ACUDIDO A LA JURISDICCIÓN DE PAZ? } & \text { TOTAL } & \text { PORCENTAJE } \\ \text { SI } & 43 & 20.7 \% \\ \text { NO } & 164 & 79.3 \%\end{array}$

Del porcentaje que ha acudido a la jurisdicción de paz para resolver conflictos, el $83.7 \%$ consideró que la intervención del juez en dicho conflicto le ayudó (para mayor ilustración ver tabla 9); respecto al trámite el $44.1 \%$ manifestó que el trámite fue normal, mientras que para el $23.2 \%$ dicho proceso fué corto (tabla 10). Por otra parte, el $88.3 \%$ de los encuestados manifestaron que las decisiones acordadas se cumplieron (tabla 11). 
Tabla 9. ¿Considera que la intervención del juez le ayudó)

$\begin{array}{lcc}\text { ¿CONSIDERA QUE LA INTERVENCIÓN DEL JUEZ LE AYUDÓ? } & \text { TOTAL } & \text { PORCENTAJE } \\ \text { SI } & 36 & 83.7 \% \\ \text { NO } & 7 & 16.2 \%\end{array}$

Tabla 10. El trámite fué

EL TRÁMITE FUE:

NORMAL

CORTO

LARGO

MUY CORTO

EXAGERADO
TOTAL

19

10

8

5

1

TOTAL

38

5
PORCENTANE

$88.3 \%$

$11.6 \%$

\section{DISCUSIÓN \\ Y CONCLUSIONES}

Durante el desarrollo de la presente investigación, se aplicaron diversos instrumentos (entrevistas, encuestas, análisis bibliográfico, análisis de las actas de conciliación y de los fallos), los cuales permitieron llevar a cabo una serie de actividades que dejaron entrever la situación actual de la justicia de paz en la ciudad de Neiva, y establecer que:

a) De acuerdo con los resultados encontrados, la jurisdicción de paz fue implementada en el año 2004 por la Alcaldía de Neiva en conjunto con el Consejo Seccional de la Judicatura, órgano encargado de la vigilancia y control de esta figura. Estos entes realizaron diferentes eventos en las distintas comunas de la ciudad para lograr el reconocimiento del juez de paz como actor principal de este mecanismo de solución de conflictos. Previo a estas estrategias pedagógicas, se brindó capacitación a los aspirantes a ejercer este cargo. Así mismo, se informó a los diferentes medios de comunicación tanto radiales como escritos para que difundieran las características de esta nueva figura y de esta manera se pudiera establecer una buena acogida entre la sociedad. Seguidamente se llevaron a cabo las elecciones de los Jueces de Paz y de Reconsideración en cada comuna, tomando posesión quienes fueron elegidos en el año 2005.

Sin embargo, a partir de este momento, la Alcaldía del municipio de Neiva comenzó a desentenderse de sus deberes para con la figura, por lo que no volvió a realizar campañas de divulgación de los jueces de paz que permitieran la consolidación de la misma, para que la mayoría de los miembros de la comunidad tuvieran conocimiento de la función que llevan a cabo en la solución de conflictos, y la importancia en la búsqueda de la paz y la armonía dela sociedad.

b) Se pudo apreciar que en las comunas 6,9 y 10 , se presenta el nivel más alto de concurrencia a los jueces de paz, debido a que quienes habitan en estos sectores son personas con grandes dificultades económicas, y en estas comunas existen algunas zonas vulnerables. Estas situaciones ponen de manifiesto un alto grado de conflictividad, siendo este un escenario de arduo trabajo para los jueces de paz, como actores transformadores de la problemática cotidiana. Por 
el contrario existen otras zonas de la cuidad donde se presenta poco nivel de concurrencia a los jueces de paz, situación que se puede atribuir a diversos factores entre los cuales se pueden mencionar algunos como: la persistencia del desconocimiento de esta figura en la población neivana, la desconfianza que genera este mecanismo en la percepción de algunos habitantes debido al carácter informal del mismo, y también se puede agregar que una alto porcentaje de los ciudadanos prefieren acudir a las instancias judiciales.

La mayoría de los casos que son llevados al conocimiento de los jueces de paz terminan en conciliación. Son muy escasos los que concluyen con fallo en equidad, lo que demuestra que el juez de paz actúa como mediador para acercar a las partes en la búsqueda de una solución que rescinda de la mejor manera el conflicto que llevó a recurrir a esta figura.

De igual manera, quienes ejercen como jueces de paz y de reconsideración, han manifestado la ausencia de apoyo logístico, económico y de capacitación por parte de las instituciones encargadas de la preparación y supervisión de ésta jurisdicción, debido que no cuentan con un espacio en el cual puedan atender a la comunidad, ya que carecen de papelería necesaria para llevar un registro detallado de los casos atendidos y de las actas de conciliación, e inclusive deben valerse de recursos propios para transportarse al lugar del conflicto cuando el caso lo amerita, lo que dificulta el eficaz cumplimiento de sus funciones.

Por lo anterior, se considera que el ánimo de servicio hacia la comunidad, la idoneidad y reconocimiento del juez de paz, como presupuestos fundamentales de esta jurisdicción no son excluyentes con la necesidad imperante de dar un auxilio económico a la labor desempeñada, que por otra parte garantizaría que los jueces de paz, no sean objeto de ofrecimiento de algunas prebendas por parte de quienes buscan aprovechar su estado de necesidad, con el fin de obtener una decisión parcializada, constituyéndose en posibles focos de corrupción al interior de esta figura alternativa.

c) Los resultados obtenidos con la implementación y puesta en funcionamiento de la jurisdicción de paz en el municipio de Neiva no han sido los esperados, debido a que es una figura desconocida por la mayoría de la ciudadanía, ya que no cuentan con el suficiente apoyo por parte de la Alcaldía Municipal, y del Consejo Seccional de la Judicatura (entes estatales que actúan de manera separada, en el manejo de la poca información que poseen.) El problema sobre el mal funcionamiento de la jurisdicción de paz en Neiva no debe recaer solo en manos de las entidades estatales que tienen a cargo el desarrollo de esta, pues si bien es cierto que su implementación y buena prestación del servicio corre por cuenta del Estado a través del Consejo Superior de la Judicatura y del Ministerio del Interior y de justicia, de igual manera el desarrollo y subsistencia de la figura, depende de la aceptación y el apoyo que las comunidades brinden a la justicia de paz para que logre convertirse en la "base del sistema judicial en Colombia" (AMAYA), pues pese a que existen ciertas criticas negativas hacia la figura al afirmarse que "Los jueces de paz son un invento del gobierno pa estratificar la justicia entre ricos y pobres" "Los jueces de paz son para descongestionar el aparato judicial y privatizar la justicia", éstas son solo especulaciones de personas que no van más allá de lo que ven, ni examinan los aspectos positivos de la figura.

Pese a las anteriores afirmaciones, se considera realizar una evaluación de la necesidad que tiene la sociedad de acudir a esta figura; lo que permitiría pensar en dos cosas: Por un lado si las personas se han visto en la necesidad de acudir a un ente estatal para que les ayuden a dar solución a sus conflictos pero se abstienen de hacerlo porque consideran que ese tipo de justicia no funciona, entonces aqui se podría decir que hay una falla en la prestación del servicio ya sea por una mala implementación, o por la carencia de herramientas para que la justicia de paz cumpla con sus

${ }^{2}$ Según lo manifestó el señor Juez de Paz Humberto Tejada Suárez, en entrevista del mes de Septiembre del año 2008. ${ }^{3}$ Ibídem. 
objetivos; dando razones a la comunidad para desconfiar de esta justicia y con ello la deje de ver como una alternativa para la solución de sus problemas.

Por otro lado, se encuentra el supuesto de que las personas no acudan a los diversos entes judiciales porque no se han visto en la necesidad de hacerlo, lo que permite deducir, que estaria en marcha la construcción de una sociedad que puede ser reconocida por sus valores y buenas costumbres, $y$ no por el auge de conflictividad que habría en ella.

A partir de estos supuestos es que se puede realizar un juicio de valor acerca de la importancia de la figura dentro de los diversos contextos sociales del país. Es por ello que no puede dejarse que la justicia de paz sufra mas deterioro, es hora de darle la importancia que se merece y la divulgación necesaria para que la jurisdicción de paz pueda posesionarse como un verdadero mecanismo alternativo de solución de conflictos que goce de la suficiente credibilidad y aceptación por parte de la sociedad, partiendo de su conocimiento, y se logre la utilización de sus servicios.

Sin duda alguna, son dos los problemas mas críticos que afronta la Jurisdicción Especial de Paz en Neiva; el primero obedece al alto nivel de desconocimiento existente entre las comunidades sobre el alcance y funciones que desempeña el Juez de paz, pues en algunos casos las personas que habitan en zonas aledañas donde ejercen las labores estos actores comunitarios no lo reconocen llegando a confundirlo con otra figura jurídica como la Ley de Justicia y Paz. La segunda dificultad no menos relevante que la anterior, y consiste en que el pequeño porcentaje que reconoce y utiliza esta jurisdicción para obtener una resolución de sus conflictos, quienes terminan afirmando que la intervención en la solución del conflicto fue buena y útil, sin embargo resaltan que existen aspectos negativos relevantes que van rezagando poco a poco la vigencia de la Jurisdicción de Paz, entre los que se encuentran principalmente la escasez de recursos para el funcionamiento y desarrollo de las diligencias conciliatorias, situación que conlleva a disminuir la presencia de habitantes ante el Juez de Paz para resolver sus conflictos.

Se sugiere replantear este tema y otros de igual envergadura, que permitan rescatar este mecanismo alternativo de solución de conflictos a nivel nacional, a través de una reforma a la normatividad vigente, a la adopción de otras medidas por parte del Consejo superior de la Judicatura y sus Consejos Seccionales a nivel local, que permitan una mejor capacitación a los jueces de paz y un mayor conocimiento de la figura en todas las comunidades del territorio Nacional. Pues como lo afirma el profesor Ardila:

"Mal se haría si un instrumento para muchas comunidades, se lleva de manera abrupta a todos los escenarios locales. Aún cuando lo que se tiene de la justicia de paz es apenas un croquis que podrá llenarse con la historia y las dinámicas especificas de las comunidades, una implementación indiscriminada podría tener efectos negativos. Es por ello que los retos que plantea la Ley 497 tendrán que asumirse con mucha responsabilidad por parte de los actores encargados de llevar a la realidad la herramienta de los jueces de paz. (Ardila; 1999:36)" 


\section{BIBLIOGRAFÍA}

- Abella de Fierro Martha Cecilia y Vargas OrtizAlfredo. (2002), "Justicia de paz y solución de los conflictos cotidianos en Neiva, Ibagué y Florencia", Editorial Universidad Surcolombiana.

- Ardila Amaya, Edgar. (1999) La Ley de Jueces de Paz en Colombia: de la norma la realidad. En: Justicia y Desarrollo. Debates. Bogotá: Corporación Excelencia en la Justicia.

-Ardila Amaya, Edgar. (1999) Justicia Comunitaria: Participación en la construcción de paz. En: Los jueces de paz: una justicia para el nuevo milenio. Ediciones Escuela Ciudadana. Cali.

- Ariza Santamaría, Rosembert. (2000) Justicia comunitaria y jueces de paz, las técnicas de la paciencia. IPC, Corporación Región Medellín

- Ariza Santamaría, Rosembert. (2007) Articulo: "la jurisdicción especial de Paz: La justicia de la esperanza", Revista lusta, Universidad Santo Tomás, Bogotá.

- Borrero Garcia, Camilo y otros. (2003) Justicia Alternativa: Estudios de Caso. CINEP. Bogotá.

Guerra Cerón, María Elena. (2005). Hacia una justicia de paz: Un asunto de interés nacional. Editora jurídica Grijley. Lima.

- Infante De la Torre, Álvaro. (2000), "Justicia Comunitaria y jueces de paz en Bolivia”. En: Norma Galves, M (editora). Justicia de Paz en la Región Andina. Experiencias comparadas, utopías compartidas. $1^{\circ}$ ed., Bogotá: Corporación Excelencia en la Justicia.

- Lovatón Palacios, David. (2000), "La justicia de paz en el Perú: aspectos positivos y límites”. En: Norma Galves, M (editora). Justicia de Paz en la Región Andina. Experiencias comparadas, utopías compartidas. $1^{\circ}$ ed., Bogotá: Corporación Excelencia en la Justicia.

- Lovatón, David y Ardito Wilfredo. (2002). Justicia de Paz: Nuevas tendencia y tareas pendientes. Instituto de Defensa Legal, Lima Perú.

- Martínez Elkin Freddy. (2005) “La Justicia de Paz, una justicia y desde los de ruana”. En: Primer Encuentro Distrital de Justicia Comunitaria y Alternativa. Bogotá, IPAZUD Edición.

- Pedroso, Joao y Trincao, Catarina. (2003), "El re-nacimiento de la Justicia de Paz: ¿una reforma democrática o tecnocrática de la justicia? Las experiencias de Italia, España, Brasil y Portugal", En: El otro Derecho, $N^{\circ} 30$, Variaciones sobre la justicia Comunitaria. Bogotá: Instituto Latinoamericano de Servicios Legales Alternativos (ILSA) y Red de Justicia Comunitaria.

- Perafan Lévano, Betsy. (1999) Algunas reflexiones sobre la ley de jueces de paz en justicia, desarrollo, Debates A.N $N^{\circ}$ 8. Corporación excelencia en lajusticia.

- Ponce Silen, Carlos Eduardo. (2000). “Ley Orgánica de la Justicia de Paz en Venezuela: ¿Impulso o freno al desarrollo de la figura? En: Norma Gálvez, M (editora). Justicia de Paz en la Región Andina. Experiencias comparadas, utopías compartidas. $1^{\circ}$ ed., Bogotá: Corporación Excelencia en la Justicia.

- Sepúlveda Franco, Álvaro. (2003) Jurisdicción de paz y los conflictos comunitarios y ambientales. En: Contrastes sobre lo justo: Debates en justicia comunitaria. IPC de la Corporación de Promoción Popular. Medellín

- Uprimny Yepes Rodrigo. (2005). “Justicia Comunitaria en Bogotá: Aproximación conceptual y reflexiones sobre su sentido político". En: Primer Encuentro Distrital de Justicia Comunitaria y Alternativa. Bogotá, IPAZUDEdición.

-Vinay Rojas, Claudia. (2000) "La justicia de paz en México". En: Norma Gálvez, M (editora). Justicia de Paz en la Región Andina. Experiencias comparadas, utopías compartidas. $1^{\circ}$ ed., Bogotá: Corporación Excelencia en la Justicia.

\section{SENTENCIAS}

Colombia, Corte Constitucional, sentencias C-536 de 1995 M.P. Jaime Córdoba Triviño.

Colombia, Corte Constitucional, sentencia C-103 de 2004 M.P. Manuel José Cepeda.

Colombia, Corte Constitucional, sentencia C- 059 de 2005 M.P. Clara Inés Vargas Hernández.

Colombia, Corte Constitucional, sentencia T-796 de 2007 M.P. Jaime Córdoba Triviño

Colombia, Corte Constitucional, sentencia T- 809 de 2008 M.P. Manuel José Cepeda. 